\title{
PAPER
}

\section{A comparative study into the one year cumulative incidence of depression after stroke and myocardial infarction}

\author{
I Aben, F Verhey, J Strik, R Lousberg, J Lodder, A Honig
}

See end of article for authors' affiliations

.....................

Correspondence to: Professor Frans R J Verhey, Department of Psychiatry and Neuropsychology, Institute of Brain and

Behaviour, Maastricht

University/University

Hospital of Maastricht,

PO Box 5800, 6202 AZ

Maastricht, Netherlands:

f.verhey@np.unimaas.nl

Received 21 August 2002

In revised form

3 January 2003

Accepted

16 January 2003

Background: The high incidence of post-stroke depression has been claimed to reflect a specific, stroke related pathogenesis in which lesion location plays an important role. To substantiate this claim, post-stroke depression should occur more often than depression after another acute, life threatening, disabling disease that does not involve cerebrovascular damage.

Objectives: To compare the cumulative one year incidence of depression after stroke and after myocardial infarction, taking into consideration differences in age, sex, and the level of handicap.

Methods: In a longitudinal design, 190 first ever stroke patients and 200 first ever myocardial infarction patients were followed up for one year. Depression self rating scales were used as a screening instrument to detect patients with depressive symptoms. Major and minor depression was assessed at one, three, six, nine, and 12 months after stroke or myocardial infarction according to DSM-IV criteria, using the structured clinical interview from DSM-IV. The severity of depressive symptoms was measured with the Hamilton depression rating scale. Level of disability and handicap was rated with the Rankin handicap scale.

Results: The cumulative one year incidence of major and minor depression was $37.8 \%$ in stroke patients and $25 \%$ in patients with myocardial infarction (hazard ratio $1.6 ; p=0.06$ ). This difference disappeared after controlling for sex, age, and level of handicap. In addition, no differences were found in the severity of depressive symptoms or in the time of onset of the depressive episode after stroke or myocardial infarction.

Conclusions: Depression occurs equally often during the first year after stroke and after myocardial infarction when non-specific factors such as sex, age, and level of handicap are taken into account. Thus the relatively high incidence of post-stroke depression seems not to reflect a specific pathogenic mechanism. Further research is needed to investigate whether vascular factors play a common role in the development of depression after stroke and myocardial infarction.

$\mathrm{D}$ ifferent pathogenic models have been proposed to explain the high prevalence of post-stroke depression. The most influential model suggested a neurobiological mechanism, with the highest risk for depression in cases with a left hemispherical infarction. ${ }^{1}$ Depression in elderly people has also been related to a more general vascular pathogenesis, being associated with white matter hyperintensities and multiple silent cerebral infarctions on magnetic resonance imaging (MRI). ${ }^{2}$ In addition, non-specific mechanisms—such as personality traits and lack of social support ${ }^{3}$ - may also be involved in the pathogenesis of post-stroke depression.

The few studies that have directly compared the prevalence of depression in patients with cerebrovascular disease and non-cerebral vascular disease reported a higher prevalence in stroke patients. ${ }^{45}$ However, these studies may be criticised because of cross sectional designs, small sample sizes, depression diagnoses based on rating scale scores, and lack of control of potentially confounding variables such as age, sex, and the severity of handicap.

In this study, we compared the cumulative one year incidence of depression after stroke and myocardial infarction, after controlling for such potential confounders. Like stroke, myocardial infarction has an acute onset, is life threatening, can recur, and tends to run a chronic course with a mild to severe impact on the patient's quality of life. Moreover, stroke and myocardial infarction share similar vascular risk factors. Therefore, if the incidence of post-stroke depression differs from post-myocardial infarction depression after controlling for sex, age, and level of handicap, this may be seen as circum- stantial evidence of a difference in the pathogenesis of depression between these two diseases.

\section{METHODS \\ Patients}

One hundred and ninety consecutive patients with a diagnosis of first ever hemispheric cerebral infarct and 200 patients with a diagnosis of first ever myocardial infarct were included in the study. The patients were recruited from the emergency department of the University Hospital of Maastricht, Netherlands. This university hospital serves approximately 200000 inhabitants, being the only hospital in the Maastricht region.

Stroke was diagnosed by a neurologist according to the WHO criteria. ${ }^{6}$ The ischaemic nature of the stroke was verified by computed tomography (CT). Patients with other types of stroke (for example, recurrent stroke, haemorrhage, or brain stem infarction) were not included, in order to increase the homogeneity of the study groups. Patient data were entered into a prospective stroke registry, which has been described in detail elsewhere. ${ }^{7}$

Abbreviations: BDI, Beck depression inventory; DSM-IV, Diagnostic and Statistical Manual of Mental Disorders, 4th edition; FAST, Frenchay aphasia screening test; HADS, hospital anxiety and depression scale HAM-D, Hamilton depression rating scale; HR, hazard ratio; MMSE, mini-mental state examination; SCID-I-R, structured clinical interview from DSM-IV; SCL-90, 90 item symptom checklist 
Table 1 Demographic and outcome related characteristics of patients with stroke and myocardial infarction

\begin{tabular}{|c|c|c|c|}
\hline Variable & Stroke $(n=190)$ & $\mathrm{MI}(n=200)$ & $\mathrm{p}$ Value \\
\hline Sex (female) & $46.8 \%$ & $23.0 \%$ & $<0.001$ \\
\hline Age (years) (mean (SD)) & $68.6(11.7)$ & $60.0(10.8)$ & $<0.001$ \\
\hline Level of education & & & $<0.001$ \\
\hline Primary school & $42.2 \%$ & $25.9 \%$ & \\
\hline Junior secondary vocational education & $25.8 \%$ & $19.3 \%$ & \\
\hline Senior secondary vocational education & $19.0 \%$ & $31.5 \%$ & \\
\hline (Pre-)university education & $13.0 \%$ & $23.3 \%$ & \\
\hline Living alone & $35.7 \%$ & $18.0 \%$ & $<0.001$ \\
\hline MMSE (median, range) & 26 (16 to 30$)$ & - & - \\
\hline FAST (median, range) & 27 (9 to 30$)$ & - & - \\
\hline Barthel (mean (SD))* & $17.4(4.7)$ & $20.0(0.2)$ & $<0.001$ \\
\hline Rankin (mean (SD)) $\dagger$ & $2.4(1.2)$ & $1.2(0.7)$ & $<0.001$ \\
\hline Personal history of depression & $21.8 \%$ & $12.0 \%$ & 0.01 \\
\hline Family history of depression & $20.1 \%$ & $10.0 \%$ & 0.006 \\
\hline Family history of other psychiatric disorders & $43.3 \%$ & $11.5 \%$ & $<0.001$ \\
\hline
\end{tabular}

Myocardial infarction was diagnosed by a cardiologist on the basis of clinical presentation, electrocardiography typical of acute myocardial infarction, and a maximum value of aspartate aminotransferase of $>80 \mathrm{U} / \mathrm{l}$ (twice the upper limit of normal). ${ }^{8}$

Patients from both cohorts were excluded for the following reasons:

- if they had a major psychiatric disorder other than affective disorders (for example, schizophrenia or a current psychotic episode);

- if they had reported a depressive episode in the weeks before the time of the stroke/myocardial infarction;

- if they had a comorbid intracerebral disease;

- if the clinician judged that they were unable to understand the informed consent procedure (for example, because of severe aphasia or dementia) after having administered the mini-mental state examination (MMSE) and the Frenchay aphasia screening test (FAST) (see Methods).

All participants gave written informed consent. The study was approved by the local medical ethics committee.

\section{METHODS}

Optimal comparability was achieved by using a similar study design and similar methods for both patient groups. All patients were followed up during the first year after stroke or myocardial infarction. One month after the stroke or myocardial infarct, all patients were interviewed using the structured clinical interview of DSM-IV (SCID-I-R), ${ }^{9}$ allowing for a diagnosis of both major and minor depression. ${ }^{10}$ The severity of depressive symptoms was measured with the 17 item Hamilton depression rating scale (HAM-D). ${ }^{11}$ All interviews were administered by well trained research physicians (IA or JS).

At the follow up interviews at three, six, nine, and 12 months, depression was diagnosed using a two step procedure. First, three psychiatric self rating scales for depression were administered-namely, the Beck depression inventory $(\mathrm{BDI}){ }^{12}{ }^{13}$ the hospital anxiety and depression scale (HADS), ${ }^{14}{ }^{15}$ and the 90 item symptom checklist (SCL-90). ${ }^{16}{ }^{17}$ Then patients who exceeded the previously defined cut off value on at least one of these scales were reinterviewed using the SCID and HAM-D. For the BDI, the cut off value was 9/10, for the HADS $7 / 8$ for both subscales separately, and for the depression subscale of the SCL-90 a threshold of 22/23 was used for men and 27/28 for women. The sensitivity of this screening procedure has previously been proven to be high, at
$91.2 \%$ in stroke patients and $85.7 \%$ in myocardial infarction patients. The specificity rates were $48.2 \%$ and $65.7 \%$, respectively. ${ }^{18} 19$

At the one month follow up, disability and handicap were rated using the Barthel index ${ }^{20}$ and Rankin score, ${ }^{21}$ respectively. The Rankin score is derived from an ordinal six point scale in which two consecutive scores can be considered to represent equal increases in the level of handicap. Data concerning demographics, level of education, living situation, family history of psychiatric disorders, and drug treatment were collected by interview. A family history of psychiatric disorders was rated separately for different groups of disorders (affective, schizophrenic, substance abuse, and so on) but was dichotomised into positive or negative psychiatric family history in order to optimise the statistical power. The personal history of depression was measured using the SCID-I-R. In stroke patients, MMSE $^{22}$ and FAST $^{23}$ were administered to measure global cognitive functioning and aphasia, respectively.

\section{Analysis}

Cumulative incidence rates for post-stroke depression and post-myocardial infarction depression were analysed using survival analysis techniques. If data on depressive status were missing, patients were considered "not depressed" if they were not depressed at the previous assessment and if the depressive state at the next assessment was validly measured. In all other situations, the case was excluded from further analysis from the time point of the missing value onward. A Cox regression analysis was undertaken, with cohort (stroke or myocardial infarction) as the independent variable, controlling for sex, age, and level of handicap (Rankin score). The output of the Cox analysis was checked for instability by influential cases and for violation of both the proportional hazards assumption and the assumption of linearity of effects.

For comparisons between descriptive sample characteristics, Student's $t$ test was used in the case of continuous normally distributed variables. The Mann-Whitney $U$ test was used for non-normally distributed or ordinal variables. A $\chi^{2}$ test was used for all dichotomous variables. Lastly, one way analysis of variance (ANOVA) was used to compare differences in HAM-D scores between major depressed, minor depressed, and non-depressed patients.

For all analyses, the level of statistical significance was set at p $<0.05$ (two tailed).

\section{RESULTS}

The stroke patients were recruited from a total sample of 444 consecutive patients with a first ever hemispheric cerebral 
Table 2 Cumulative incidence of depression after stroke or myocardial infarction during one year of follow up (irrespective of the distribution of age, sex, and handicap)

\begin{tabular}{llllll}
\hline & $\begin{array}{l}\text { Time of } \\
\text { assessment } \\
\text { (months) }\end{array}$ & $\begin{array}{l}\text { Number at } \\
\text { risk }\end{array}$ & $\begin{array}{l}\text { Incident cases of } \\
\text { depression }\end{array}$ & $\begin{array}{l}\text { Proportion } \\
\text { depressed }\end{array}$ & $\begin{array}{l}\text { Cumulative proportion } \\
\text { depressed }\end{array}$ \\
\hline Stroke & 1 & 190 & 41 & $21.6 \%$ & $21.6 \%$ \\
& 3 & 137.5 & 7 & $5.1 \%$ & $25.6 \%$ \\
& 6 & 117 & 7 & $6.0 \%$ & $30.0 \%$ \\
& 9 & 107 & 6 & $5.6 \%$ & $33.9 \%$ \\
& 12 & 98 & 7 & $7.1 \%$ & $38.7 \%$ \\
MI & 1 & 200 & 28 & $14.0 \%$ & $14.0 \%$ \\
& 1 & 166 & 9 & $5.4 \%$ & $18.7 \%$ \\
& 3 & 151 & 4 & $2.6 \%$ & $20.8 \%$ \\
& 6 & 146.5 & 11 & $7.5 \%$ & $26.8 \%$ \\
& 9 & 134.5 & 3 & $2.2 \%$ & $28.4 \%$ \\
\hline
\end{tabular}

$\mathrm{Ml}$, myocardial infarction.

Table 3 Cox regression analysis on the effect of cohort (stroke or myocardial infarction) on the cumulative incidence of depression (major and minor together) with and without controlling for sex and the interaction between age and level of handicap

\begin{tabular}{lllll}
\hline & & HR & $95 \% \mathrm{Cl}$ & $\mathrm{p} \mathrm{Value*}$ \\
\hline Bivariate model & Stroke cohort & 1.42 & 0.99 to 2.02 & 0.06 \\
& & & \\
Multivariate model & Stroke cohort & 1.08 & 0.70 to 1.67 & 0.72 \\
& Female sex & 1.59 & 1.08 to 2.34 & 0.02 \\
& Age & 1.02 & 0.99 to 1.06 & 0.18 \\
& Rankint & 2.05 & 0.99 to 4.23 & 0.05 \\
& Age $\times$ Rankin & 0.99 & 0.97 to 0.996 & 0.007
\end{tabular}

*2 tailed level of significance.

tRankin scores were inverted in order to create an interaction term that increases with increasing age and/or decreasing level of handicap.

$\mathrm{Cl}$, confidence interval; $\mathrm{HR}$, hazard ratio.

infarction. One hundred and ninety three patients (43.5\%) were excluded, for the following reasons: severe comorbidity with severe cognitive disorders, including aphasia ( 54 patients $(28.0 \%))$; severe somatic comorbidity, for example loss of consciousness, general frailty, and comorbid intracerebral disease (38 patients $(19.7 \%)$ ); and combined cognitive and somatic comorbidity ( 12 patients $(6.2 \%)$ ). Further reasons for exclusion were death within one month after stroke (37 patients, $19.2 \%)$, current depressive episode at the time of the stroke (eight patients, $4.1 \%$ ), and current other major psychiatric disorder (four patients, 2.1\%). The remaining 40 patients $(20.7 \%)$ were excluded for other reasons (living outside the Maastricht region, intractability despite repeated efforts, language barrier, and so on). Of the remaining 251 eligible patients, $61(24.3 \%)$ refused participation. Thus, 190 stroke patients remained in the study.

In the myocardial infarction group, 200 of a total of 415 consecutive patients were included $(48 \%)$. Ninety five patients were excluded $(22.9 \%)$. Reasons for exclusion were death within the first month after the infarct (28 patients, $29.5 \%$ ), severe comorbidity (19 patients, 20.0\%), and psychiatric disorder (six patients $(6.3 \%$ ) had depressive disorder at the time of the myocardial infarct, and three $(3.2 \%)$ had some other major psychiatric disorder). The remaining 39 patients $(41.1 \%)$ were excluded for reasons such as living outside the Maastricht region, intractability despite repeated efforts, language barrier, and so on. Of the 320 eligible patients, 120 $(37.5 \%)$ refused participation.

Patients who refused to participate were somewhat older than the participants in both the stroke cohort (mean (SD): 72.4 (9.7) $v 68.6(11.7)$ years; $t(2)=2.6, p=0.01)$ and the myocardial infarction cohort (65.6 (12.2) v 60.1 (10.8) years; $t(2)=4.2, \mathrm{p}<0.001)$. In the myocardial infarct cohort, women refused to participate more often than men $(53.1 \% \mathrm{v}$ $\left.30.6 \% ; \chi^{2}(1)=14.6 ; p<0.001\right)$. Refusers were all asked at least to fill out the SCL-90 and HADS, and $57.4 \%$ of the stroke patients and $33.3 \%$ of the myocardial infarction patients did so; in both cohorts, the mean depression scores of refusers were comparable with the scores of those who participated.

Follow up data were incomplete in 33 of the 190 stroke patients who participated in the study $(17.4 \%)$, in whom depression had not been diagnosed, as compared with 14 of the 200 patients in the myocardial infarction group (7.0\%).

The major characteristics of the two study samples are summarised in table 1 . The stroke cohort differed from the myocardial infarction cohort with regard to age, sex distribution, level of education, and level of handicap. On average, stroke patients were almost nine years older than the patients with myocardial infarction. In the stroke cohort, $47 \%$ were women, as opposed to $23 \%$ in the myocardial infarction cohort. Stroke patients were more severely disabled and handicapped.

The one year cumulative incidence of depression (major and minor combined) was $38.7 \%$ after stroke and $28.4 \%$ after myocardial infarction (adjusted for cases with incomplete follow up; see table 2). Thus stroke patients tended to have a higher risk of depression than patients with myocardial infarction (hazard ratio (HR) $1.4 ; \mathrm{p}=0.06$ ). However, if sex, age, level of handicap (Rankin score), and the interaction between younger age and greater severity of handicap (age*Rankin) were entered into the analysis as covariates, this tendency disappeared (HR 1.08; $p=0.72$ ) (fig l). Moreover, both female sex (HR 0.6, p = 0.02) and age*Rankin (HR 0.9, $\mathrm{p}=0.007$ ) independently predicted a depressive outcome 


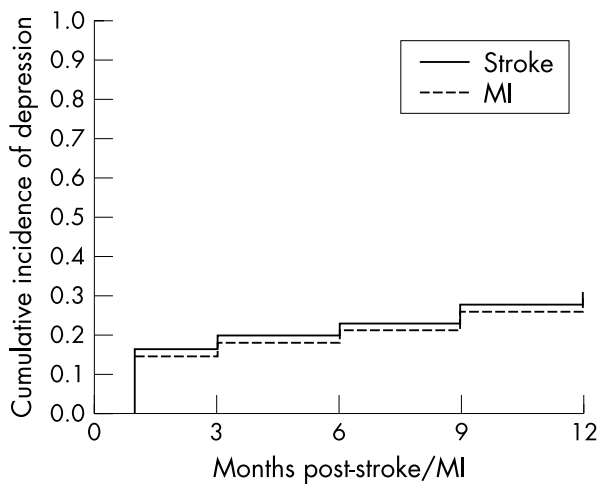

Figure 1 Cumulative one year incidence of depression after stroke or myocardial infarction (MI) adjusted for covariates (age, sex, level of handicap, and age ${ }^{*}$ level of handicap).

(table 3). When major depression was analysed separately, its cumulative incidence was significantly higher after stroke than after myocardial infarction on bivariate analysis (HR 2.1; $\mathrm{p}=0.003$ ), but again, this difference disappeared after controlling for sex and age*Rankin (HR 1.36; $\mathrm{p}=0.31$ ).

In both cohorts about half of the incident cases of depression had already developed by one month $(50.7 \%$ of depressed stroke patients and $46.7 \%$ of depressed myocardial infarction patients; table 2), indicating that depression had an early onset in many patients. In the stroke cohort, 41 patients $(23.3 \%)$ met DSM-IV criteria for major depressive disorder and $27(15.4 \%)$ met criteria for minor depressive disorder. In the myocardial infarction cohort, 23 patients (11.9\%) were diagnosed with major depression and 32 (16.5\%) with minor depression. No difference in the severity of depressive symptoms was found between stroke and myocardial infarction cohorts, nor did the difference in HAM-D scores for major, minor, and non-depressed patients differ between the two cohorts.

Of the patients who were considered not to be depressed, $7.6 \%$ in the stroke cohort and $6.5 \%$ in the myocardial infarction cohort were prescribed antidepressants by their general practitioner at some time during follow up. The reason for taking this treatment was not known. Reanalysis of the cumulative incidence of depression in both cohorts when considering these patients as depressed did not yield different results (data not shown).

\section{DISCUSSION}

To our knowledge, this study is the first to compare directly the cumulative one year incidence of depression in patients after stroke or after myocardial infarction, using a similar longitudinal study design, setting, and methods. After we controlled for differences in age, sex, and level of handicap between stroke patients and patients with myocardial infarction, there was no longer a statistically significant difference in the incidence of depression between the two cohorts. In both groups the highest incidence of depression was found in the early phase (that is, one month) after the stroke or myocardial infarct.

At first sight, the results of our study support the general impression from published reports that the incidence of depression is higher in stroke patients than in patients with other vascular diseases. Our data do not, however, favour the hypothesis of a specific relation between depression and cerebral infarction, as the incidence of depression after stroke did not differ from the incidence after myocardial infarction after controlling for confounding variables. Thus the increased incidence of depression after stroke and myocardial infarction may reflect other factors shared by the two conditions. One possible mechanism is that generalised damage to the vascular system in the brain may subsequently affect mood regulatory processes - the so called vascular hypothesis, as has been put forward by Krishnan and coworkers. ${ }^{2}$ This more general vascular mechanism could play a role in both myocardial infarction and stroke. We could not test this because brain imaging data on vascular brain damage were not available for the myocardial infarction cohort, although there is some indirect evidence to support the hypothesis. Several vascular risk factors-such as hypertension, raised serum cholesterol, and diabetes mellitus - that are common to both stroke and myocardial infarction, have also been related to depression. ${ }^{24}{ }^{25}$ In addition, damage to the small vessels of the brain, measured as white matter hyperintensities on MRI ${ }^{26}$ and silent cerebral infarcts ${ }^{27}{ }^{28}$ have also been related to depression. However, less specific mechanisms may be involved as well. In line with the general diathesis-stress model, both stroke and myocardial infarction can be considered as powerful stressors, with often drastic consequences for a patient's physical, psychological, and social functioning. Other factors that influence vulnerability, such as a history of depression and personality traits, ${ }^{29}$ may also be involved. The complex interacting effects of physical illness with psychological and social factors may account for a large part of the variance in mood dysregulation in physically ill patients.

Our study has various strengths, such as its prospective design and the use of well defined assessment scales and methods. A drawback, however, is that patients with severe cognitive dysfunction (dementia, aphasia) or general frailty were excluded. Thus the results cannot be generalised to patients with severe comorbid conditions, commonly found in geriatric patients. In the stroke cohort in particular, this may have led to underrepresentation of patients with severe disabilities. As we found a more severe level of handicap to be predictive of depression, we cannot rule out the possibility that the true incidence of post-stroke depression would be higher in less selected cohorts.

\section{Conclusions}

We found no evidence that the incidence of depression is higher in stroke patients than in patients with noncerebrovascular disease, after taking confounding factors such as age, sex, and the level of handicap into account. This challenges the hypothesis that specific cerebral factors play a major role in the pathogenesis of post-stroke depression.

\section{Authors' affiliations}

I Aben, F Verhey, J Strik, R Lousberg, A Honig, Department of Psychiatry and Neuropsychology, Institute Brain and Behaviour, University of Maastricht, Maastricht, Netherlands

J Lodder, Department of Neurology, Institute Brain and Behaviour, University of Maastricht

Competing interests: none declared

\section{REFERENCES}

1 Robinson RG, Kubos KL, Starr LB, et al. Mood changes in stroke patients: relationship to lesion location. Compr Psychiatry 1983;24:555-66.

2 Krishnan KR, Hays JC, Blazer DG. MRI-defined vascular depression. Am J Psychiatry 1997; 154:497-501.

3 Morris PL, Robinson RG, Raphael B, et al. The relationship between the perception of social support and post-stroke depression in hospitalized patients. Psychiatry 1991;54:306-16.

4 Rao R, Jackson S, Howard R. Depression in older people with mild stroke, carotid stenosis and peripheral vascular disease: a comparison with healthy controls. Int J Geriatr Psychiatry $2001 ; 16: 175-83$.

5 Murphy MT. The epidemiology of depression in acute stroke and cardiac in-patients. Pennsylvania: The Pennsylvania State University, 1996. [PhD thesis.]

6 NINDS. Special report from the National Institute of Neurological Disorders and Stroke. Classification of cerebrovascular diseases III. Stroke 1990;21:637-76. 
7 Boon A, Lodder J, Heuts-van Raak L, et al. Silent brain infarcts in 755 consecutive patients with a first-ever supratentorial ischemic stroke. Relationship with index-stroke subtype, vascular risk factors, and mortality. Stroke 1994;25:2384-90.

8 Pasternak RC, Braunwald E, Sobel RE. Acute myocardial infarction. In: Braunwald E, ed. Heart disease: a textbook of cardiovascular medicine, 4th ed. Philadelphia: WB Saunders Co, 1992:1200-91

9 First MB, Spitzer RL, Gibbon M, et al. Structured clinical interview for DSM-IV axis I disorders - patient edition (SCID-I/P, version 2.0). New York: Biometrics Research Department, New York State Psychiatric Institute, 1995.

10 American Psychiatric Association. Diagnostic and statistical manual of mental disorders, 4th ed. Washington, DC: American Psychiatric Press, 1994

11 Hamilton M. A rating scale for depression. J Neurol Neurosurg Psychiatry 1960;23:56-62.

12 Beck AT, Ward CH, Mendelson M, et al. An inventory for measuring depression. Arch Gen Psychiatry 1961;4:561-71.

13 Richter $\mathbf{P}$, Werner J, Heerlein A, et al. On the validity of the Beck depression inventory. A review. Psychopathology 1998:31:160-8.

14 Zigmond AS, Snaith RP. The hospital anxiety and depression scale. Acta Psychiatr Scand 1983;67:361-70.

15 Herrmann C. International experiences with the hospital anxiety and depression scale - review of validation data and clinical results. Psychosom Res 1997:42:17-41.

16 Derogatis LR, Lipman RS, Covi L. SCL-90: an outpatient psychiatric rating scale - preliminary report. Psychopharmacol Bull 1973;9:13-27.

17 Arrindell WA, Ettema JHM. Dimensionele structuur, betrouwbaarheid en validiteit van de Nederlandse bewerking van de Symptom Checklist (SCL-90). Nederlands Tijdschrift voor Psychologie 1981;43:381-7. [In Dutch.]
18 Aben I, Verhey F, Lousberg R, et al. Validity of the Beck depression inventory, hospital anxiety and depression scale, SCL-90, and Hamilton depression rating scale as screening instruments for depression in stroke patients. Psychosomatics 2002;43:386-93.

19 Strik J, Honig A, Lousberg R, et al. Sensitivity and specificity of observer and self-report questionnaires in major and minor depression following myocardial infarction. Psychosomatics 2001;42:423-8.

20 Mahoney FI, Barthel DW. Functional evaluation: the Barthel index. State Med J 1965;14:61

21 Rankin J. Cerebral vascular accidents in patients over the age of 60. 2 Prognosis. Scot Med J 1957;2:200-15.

22 Folstein MF, Folstein SE, McHugh PR. "Mini-mental state": a practica method for grading the cognitive state of patients for the clinician. $J$ Psychiatr Res 1975;12:189-98.

23 Enderby PM, Wood VA, Wade DT, et al. The Frenchay aphasia screening test: a short, simple test for aphasia appropriate for non-specialists. Int Rehabil Med 1987;8:166-70.

24 Carney RM, Rich MW, Tevelde A, et al. Major depressive disorder in coronary artery disease. Am J Cardiol 1987:60:1273-5.

25 Rabkin JG, Charles E, Kass F. Hypertension and DSM-III depression in psychiatric outpatients. Am J Psychiatry 1983;140:1072-4.

26 Krishnan KR, Goli V, Ellinwood EH, et al. Leukoencephalopathy in patients diagnosed as major depressive. Biol Psychiatry 1988:23:519-22.

27 Fujikawa T, Yamawaki S, Touhouda Y. Incidence of silent cerebral infarction in patients with major depression. Stroke 1993;24:1631-4.

28 Bryan RN, Manolio TA, Schertz LD, et al. A method for using MR to evaluate the effects of cardiovascular disease on the brain: the Cardiovascular Health Study. Am J Neuroradiol 1994;15:1625-33.

29 Aben I, Denollet J, Lousberg R, et al. Personality and vulnerability to depression after stroke: a 1-year follow-up study. Stroke 2002:33:2391-5.

\section{$\mathrm{ECHO}$}

\section{Radiotherapy can maintain sight in optic nerve sheath meningioma}

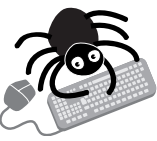

Please visit the Journal of Neurology, Neurosurgery, and Psychiatry website [www.jnnp.com] for link to this full article. atients who need treatment for primary optic nerve sheath meningioma (ONSM) may be spared the blindness that follows surgery with a treatment which has proved successful in the largest series of patients studied so far.

The study of 15 patients used stereotactic fractionated conformal radiotherapy to treat ONSM and halt its spread. At a mean follow up of 37 months (range 12-71 months) the tumour had not progressed in any patient. Sight was maintained and, indeed, vision improved in seven patients-who had larger visual field $(\geqslant 8 \%)$ - and one with greater visual acuity $(\geqslant 2$ lines). The procedure provoked only local reddening and local hair loss, which reversed with time, and hyperprolactinaemia (two patients) and partial hypophyseal insufficiency (one).

Twelve patients were women (mean age 51.5 years) and three were men (mean age 35 years). Systemic disease was excluded, and ONSM was confirmed by biopsy (three patients) or MRI or computed tomography (12). Each patient had a thorough eye examination and tests for acuity and visual fields. All patients received 54 Gy total irradiation in 28 fractions during 5.5 weeks. They were followed up closely, with eye examinations, radio-oncological and endocrine tests, and MRI scans.

Primary ONSM is rare but nearly always results in blindness-whether left alone or surgically treated when it starts to affect surrounding tissues. This alternative treatment is a major advance. The study's authors recommend it for all stages of ONSM, to stand the best chance of maintaining eyesight, though only longer follow up will tell whether the tumours eventually recur.

A British Journal of Ophthalmology 2002;86:1265-1268. 